\title{
Novel pathogenic OCRL mutations and genotype-phenotype analysis of Chinese children affected by oculocerebrorenal syndrome: two cases and a literature review
}

\author{
Yu Zhang ${ }^{1}$, Linxia Deng ${ }^{1}$, Xiaohong Chen ${ }^{2}$, Yingjie Hu${ }^{1}$, Yaxian Chen ${ }^{1}$, Kang Chen ${ }^{1}$ and Jianhua Zhou ${ }^{1 *}$ (i)
}

\begin{abstract}
Background: Oculocerebrorenal syndrome of Lowe is a rare X-linked disorder characterized by congenital cataracts, mental retardation, and proximal tubulopathy. This condition is caused by a mutation of OCRL gene (located at chromosome Xq26.1), which encodes an inositol polyphosphate 5-phosphatase.

Case presentation: We identified two novel OCRL mutations in two unrelated Chinese boys, each with a severe phenotype of Lowe syndrome. A novel de novo deletion (hemizygous c.659_662delAGGG, p.E220Vfs*29) was present in patient 1 and a novel splicing mutation (hemizygous c.2257-2A $>$ T) that was maternally inherited was present in patient 2. A renal biopsy in patient 2 indicated mild mesangial proliferative glomerulonephritis, mild focal mononuclear cells infiltration, and interstitial focal fibrosis. Moreover, renal expression of OCRL-1 protein in patient 2 was significantly reduced compared to a control patient with thin basement membrane disease.

Conclusions: This study reports two novel OCRL variants associated with severe ocular and neurologic deficiency, despite only mild renal dysfunction. Based on our two patients and a literature review, the genotype-phenotype correlation of OCRL mutations with this severe phenotype of Lowe syndrome suggest a possible clustering of missense, deletion, and nonsense mutations in the 5-phosphatase domain and Rho-GAP domain in the Chinese population.
\end{abstract}

Keywords: Proximal tubulopathy, Lowe syndrome, OCRL gene, Splicing mutation, Deletion mutation

\section{Background}

Oculocerebrorenal syndrome of Lowe (OCRL; OMIM \#309000), also known as Lowe syndrome, is an X-linked recessive multisystem disorder in which patients present with major abnormalities of the eyes (congenital cataracts), the kidneys (renal tubular acidosis, Fanconi syndrome, renal rickets), and the central nervous system (hypotonia, areflexia, and mental retardation) $[1,2]$.

\footnotetext{
*Correspondence: jhzhou99@qq.com

${ }^{1}$ Department of Pediatrics, Tongji Hospital, Tongji Medical College, Huazhong University of Science and Technology, No.1095, Jiefang Ave., Wuhan 430030, People's Republic of China

Full list of author information is available at the end of the article
}

It is a rare disease with an estimated prevalence of about $1 / 500,000$ in the general population. The manifestations of tubular dysfunction at onset vary among patients, and disease severity tends to increase with age. Proteinuria with low molecular weight (LMW) proteins is invariably present and aminoaciduria, hyperphosphaturia, bicarbonaturia, and hypercalciuria are frequently present. Progressive proximal tubulopathy ultimately leads to renal failure $[2,3]$.

This disease results from mutations of the OCRL gene (chromosome Xq26.1), which contains 24 exons and encodes a $105-\mathrm{kD}$ phosphatidylinositol $(4,5)$ biphosphate $(\mathrm{PI}(4,5) \mathrm{P} 2) 5$-phosphatase $[4,5]$. This protein is primarily 
located in the trans-Golgi network, endosomes, and endocytic clathrin-coated pits. A deficiency of this protein leads to dysregulation of vesicular transport, which may be decisive for the development of organ dysfunction that is typical of Lowe syndrome [6]. Most pathogenic mutations are deletions, frame-shifts, or premature termination codons; missense mutations and splice-site mutations are less frequent [7]. Here, we report two novel pathogenic DNA variations, a hemizygous deletion mutation c.659_662delAGGG and a hemizygous splicing mutation c.2257-2A $>\mathrm{T}$, in two boys from China who had proximal tubulopathy and congenital cataracts due to Lowe syndrome, and discuss the genotype-phenotype correlations of this disease.

\section{Case presentation}

\section{Case 1}

A 5-year-old boy was referred to our department with proteinuria, delayed motor milestones, hypotonia, congenital cataract, and failure to thrive. His past medical history indicated he was born at full term after a normal pregnancy, received surgery for congenital cataracts at the age of 3 months, and experienced several limb fractures. He was the first child of a nonconsanguineous marriage, and detailed pedigree analysis indicated no family history of congenital disorders. At birth, his weight was $3200 \mathrm{~g}(-1 \mathrm{SD}-$ Median) and length was $51 \mathrm{~cm}$ (Median- +1SD). The boy lifted his head at 8 months, sat up at 12 months, walked unassisted at 30 months, and talked at 3 years.

The physical examination at presentation indicated his body weight was $13.3 \mathrm{~kg}(<-3 S \mathrm{SD})$ and length of $96 \mathrm{~cm}$ (<-3SD). He presented with slight frontal bossing, deepset eyes, chubby cheeks, low-set ears, sparse hair, funnel chest, simian line, joint hypermobility, decreased muscle tone, and hyporeflexia. The laboratory findings indicated occasionally positive urine glucose, hypercalciuria, phosphaturia, mild proteinuria, $\beta 2$-microglobulinuria, metabolic acidosis, and occasional elevations of creatinine kinase and lactate dehydrogenase (Table 1). The estimated glomerular filtration rate (eGFR), calculated using the Schwartz formula, was $162.6 \mathrm{~mL} / \mathrm{min} / 1.73 \mathrm{~m}^{2}$. Renal size and shape were normal based on an ultrasound examination. Magnetic resonance imaging (MRI) of the brain indicated mild ventriculomegaly, lacunar lesions in the bilateral white matter consistent with periventricular leukomalacia, and multiple gliotic lesions, which were hypointense on T1-weighted and hyperintense on T2-weighted analyses (Fig. 1). An electroencephalography examination indicated no abnormalities. He was attending a school for children with special needs because his IQ was 55 and he had serious sensory integrative dysfunction.
Table 1 Laboratory values of two patients with OCRL syndrome

\begin{tabular}{|c|c|c|c|}
\hline Clinical parameter & Case 1 & Case 2 & Normal range \\
\hline \multicolumn{4}{|l|}{ Urine } \\
\hline $\mathrm{pH}$ & 7.5 & 6.5 & $4.5-8.0$ \\
\hline Glucose (quantitative) & $\begin{array}{l}\text { Occasion- } \\
\text { ally } \\
\text { positive }\end{array}$ & Negative & Negative \\
\hline Calcium (mmol/kg/24 h) & 0.19 & 0.388 & $<0.1$ \\
\hline Phosphorus (mmol/kg/24 h) & 0.87 & 0.52 & $0.5-0.6$ \\
\hline Protein (mg/24 h) & 624.7 & 226.4 & $\leq 140$ \\
\hline ß2-microglobulin (mg/L) & 65.72 & $>80$ & $<0.2$ \\
\hline \multicolumn{4}{|l|}{ Blood } \\
\hline HCO3- (mmol/L) & 15.6 & 17.7 & $22.0-29.0$ \\
\hline Calcium (mmol/L) & 2.34 & 2.60 & $2.2-2.7$ \\
\hline Phosphorus (mmol/L) & 1.53 & 1.70 & $1.05-1.8$ \\
\hline Creatinine kinase $(\mathrm{U} / \mathrm{L})$ & 274 & 62 & $\leq 190$ \\
\hline Lactate dehydrogenase (U/L) & 455 & 382 & $120-300$ \\
\hline $25(\mathrm{OH})$ vitamin $\mathrm{D}(\mathrm{ng} / \mathrm{mL})$ & 29.4 & 26.9 & $\geq 30$ \\
\hline Urea $(\mathrm{mmol} / \mathrm{L})$ & 4.28 & 2.00 & $1.7-8.3$ \\
\hline Serum creatinine ( $\mu \mathrm{mol} / \mathrm{L})$ & 22 & 22 & $59-104$ \\
\hline Uric acid $(\mu \mathrm{mol} / \mathrm{L})$ & 183.4 & 162.0 & $202.3-416.5$ \\
\hline
\end{tabular}

Whole-exome sequencing, with validation by Sanger sequencing, indicated a de novo hemizygous pathogenetic c.659_662delAGGG mutation in exon 8 of the OCRL gene (NM_000276.3; Fig. 2a), confirming the diagnosis. This deletion caused a frame shift from protein position 220 (between the $\mathrm{PH}$ and 5-phosphatase domains) leading to an insertion of 28 aberrant amino acids and a premature termination codon, resulting in a truncated protein of 247 out of 901 amino acids (p.E220Vfs*29; Fig. 2b) with an altered three-dimensional structure (Fig. 2c).

To date, he is receiving treatment with an angiotensin converting enzyme inhibitor (ACEI) for proteinuria, hydrochlorothiazide for hypercalciuria, a citrate mixture for metabolic acidosis, and rehabilitation training.

\section{Case 2}

The boy, 7 months old, was the first baby of a healthy nonconsanguineous couple. The pregnancy was uneventful, the baby had spontaneous vaginal delivery, and his birth weight was $3450 \mathrm{~g}$ (Median- + 1SD). The boy had bilateral cataracts since birth, and underwent surgery at the age of 3 months, but his visual acuity remained poor. At the age of 7 months, he could not roll over or crawl, and could not sit without support. He was admitted to our hospital with proteinuria. At admission, physical examination indicated his weight was $11 \mathrm{~kg}(+2 \mathrm{SD}-+3 \mathrm{SD})$, height was $76 \mathrm{~cm}(+2 \mathrm{SD}-+3 \mathrm{SD})$, and that he had sparse hair, chubby cheeks, bilateral nystagmus, visual impairment, 


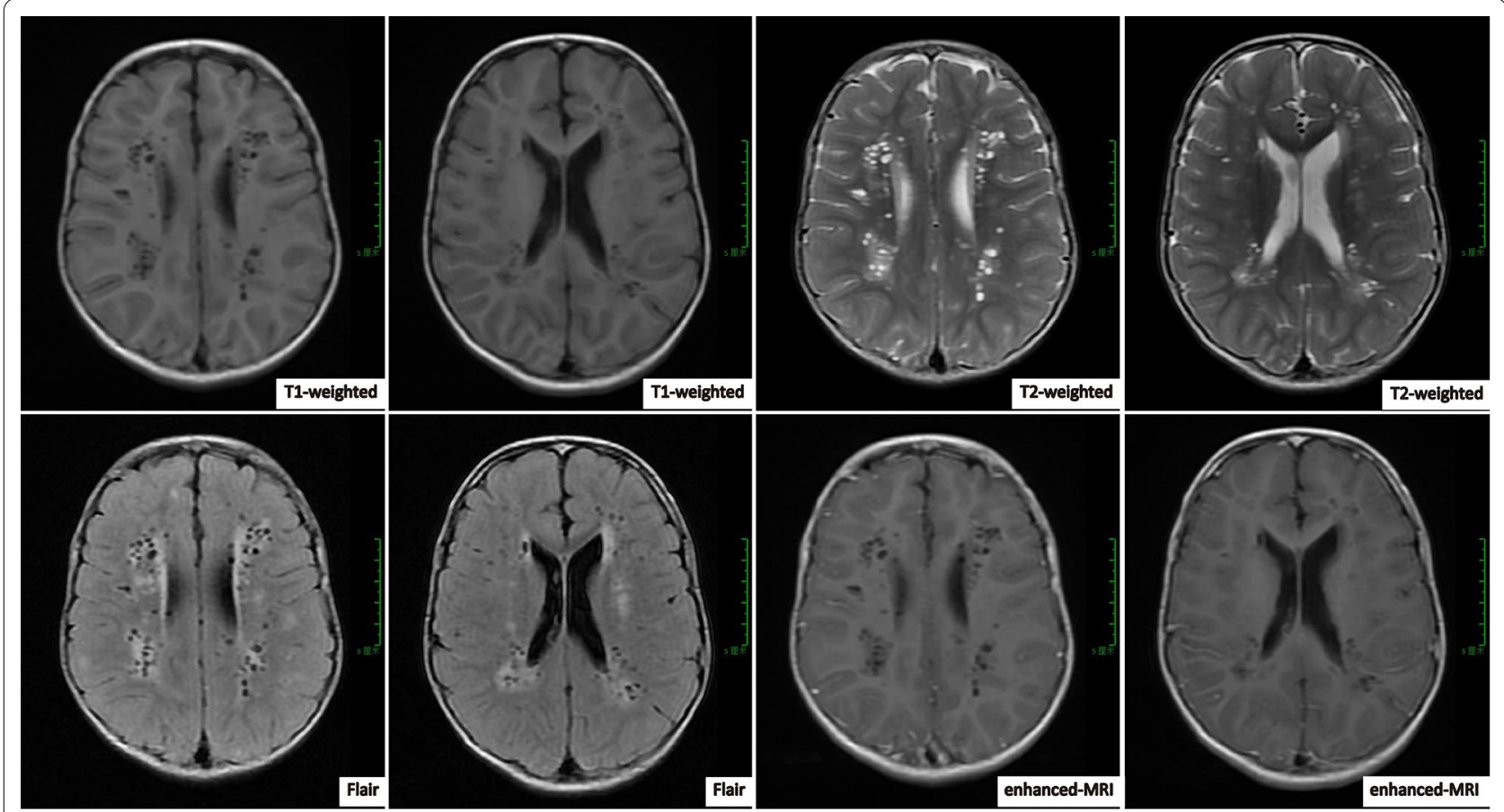

Fig. 1 Brain MRI of patient 1 at the age of five years. Note the presence of ventriculomegaly, bilateral periventricular leukomalacia, and multiple gliotic lesions

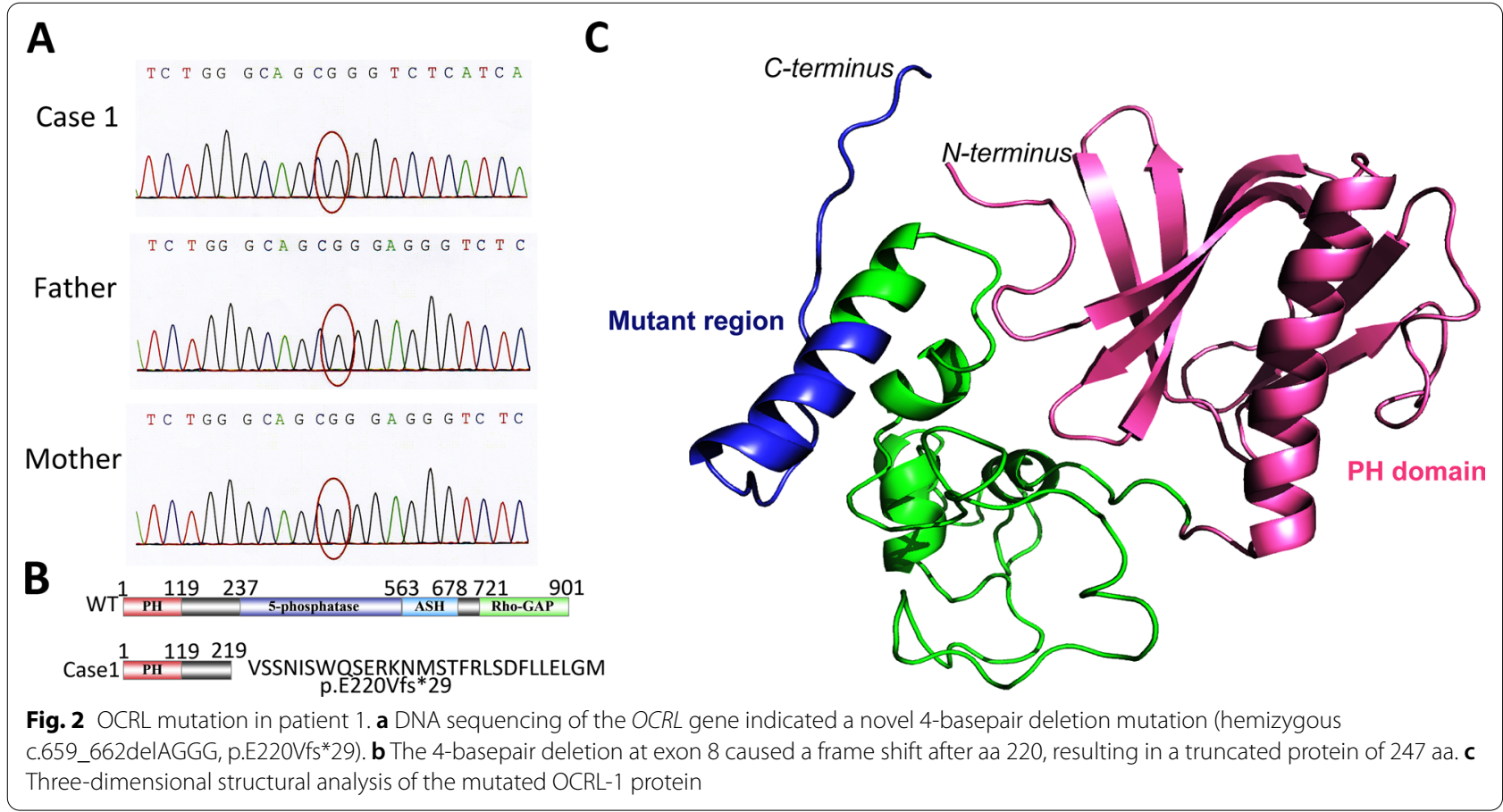

hypotonia, and absence of deep tendon reflexes. The laboratory findings indicated hypercalciuria, mild proteinuria, $\beta 2$-microglobulinuria, metabolic acidosis, elevation of lactate dehydrogenase, and hypovitaminosis D (Table 1). The eGFR (Schwartz formula) was $137 \mathrm{~mL} /$ $\min / 1.73 \mathrm{~m}^{2}$. An ultrasound examination indicated the 
size and shape of the kidneys were normal (left kidney: $6.5 \times 2.5 \mathrm{~cm}$, right kidney: $6.0 \times 2.5 \mathrm{~cm}$ ). MRI of the brain indicated severe ventriculomegaly, white matter shrinkage, leukoaraiosis, cerebellar hypoplasia, and a large cistern magna cyst (Fig. 3a). The electroencephalography results were normal. He could not complete the evaluation of psychomotor development because of poor vision. Histological examination of a renal biopsy sample indicated mild mesangial proliferation, mild infiltration of focal mononuclear cells, and interstitial focal fibrosis (Fig. 3b). Tubular lesions, including atrophy, necrosis, dilatation, and protein casts, were not detected.

To confirm the clinical diagnosis of Lowe syndrome, we analyzed the $O C R L$ gene. Whole-exome sequencing, with Sanger sequencing validation, indicated a hemizygous c.2257-2A $>\mathrm{T}$ DNA variant in $O C R L$ intron 20

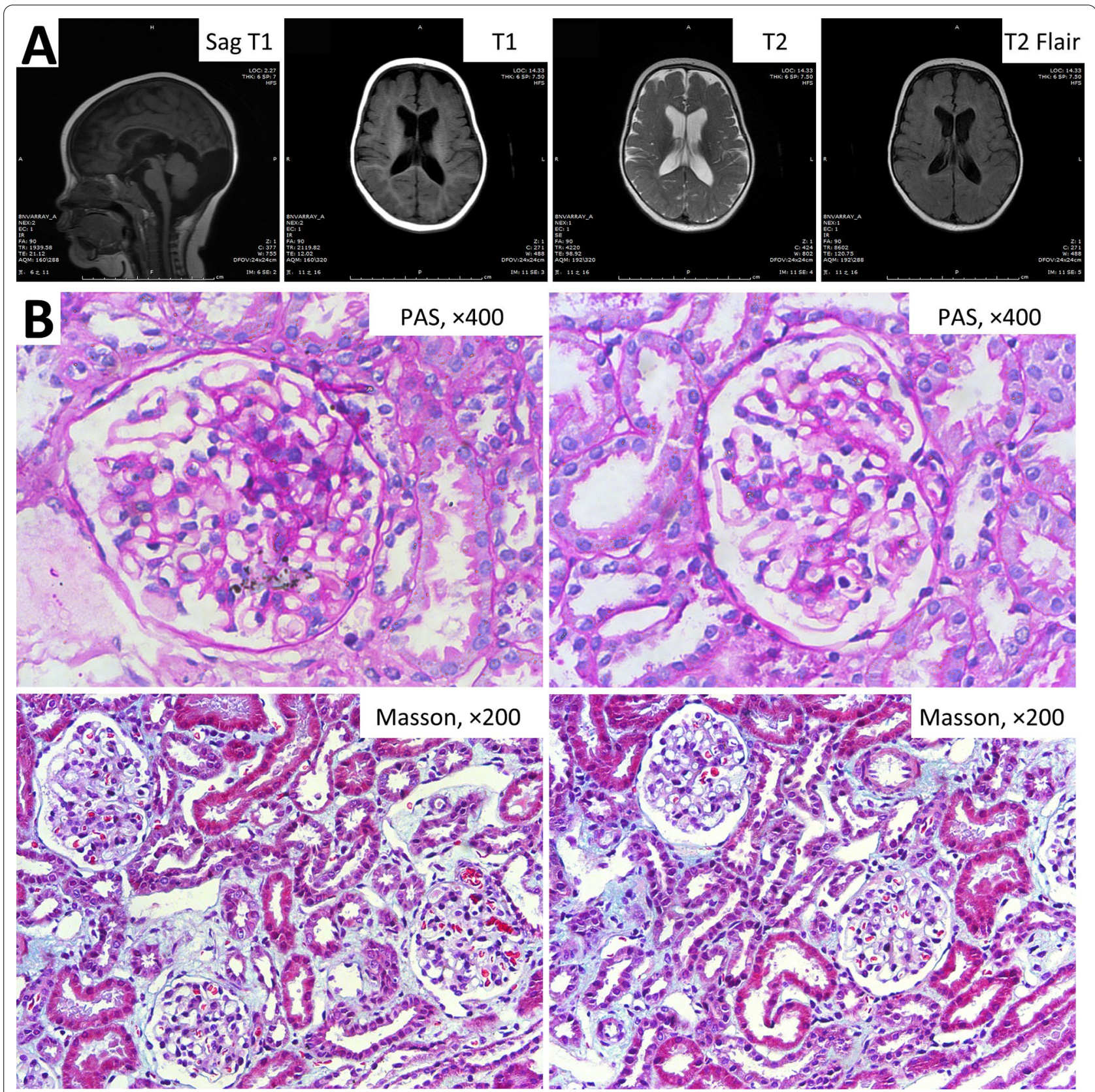

Fig. 3 Brain MRI and renal pathological changes of patient 2 at the age of 7 months. a Note the presence of ventriculomegaly, white matter shrinkage, leukoaraiosis, cerebellar hypoplasia, and a large cistern magna cyst. b Note the presence of interstitial focal fibrosis with infiltration of focal mononuclear cells and mild mesangial proliferation 
(NM_000276.3; Fig. 4a), a novel splicing variant. We then designed two pairs of primers for reverse transcription PCR of exon 8 and exon 12, but this yielded no transcriptional products. Therefore, we speculated that the mutated transcript was removed by nonsense mediated degradation (NMD) of the mRNA. We only detected the expression of OCRL-1 protein in renal tissues by immunohistochemistry using an antiOCRL polyclonal antibody against the C-terminus of the human OCRL-1 protein (Proteintech Group, USA). This patient had a markedly reduced content of OCRL-1 in kidney tissue compared with a control patient with thin membrane basement disease (Fig. 4b). Thus, this splicing mutation in the OCRL gene led to almost no expression of the wild-type protein. The patient's mother was heterozygous for the c.2257$2 \mathrm{~A}>\mathrm{T}$ mutation, but had no proximal tubulopathy, and refused an ophthalmologic examination.

We treated this patient with ACEI for proteinuria, a citrate mixture for metabolic acidosis, hydrochlorothiazide for hypercalciuria, intraocular lens implantation, and rehabilitation training to improve his motor and sensory integrative function.

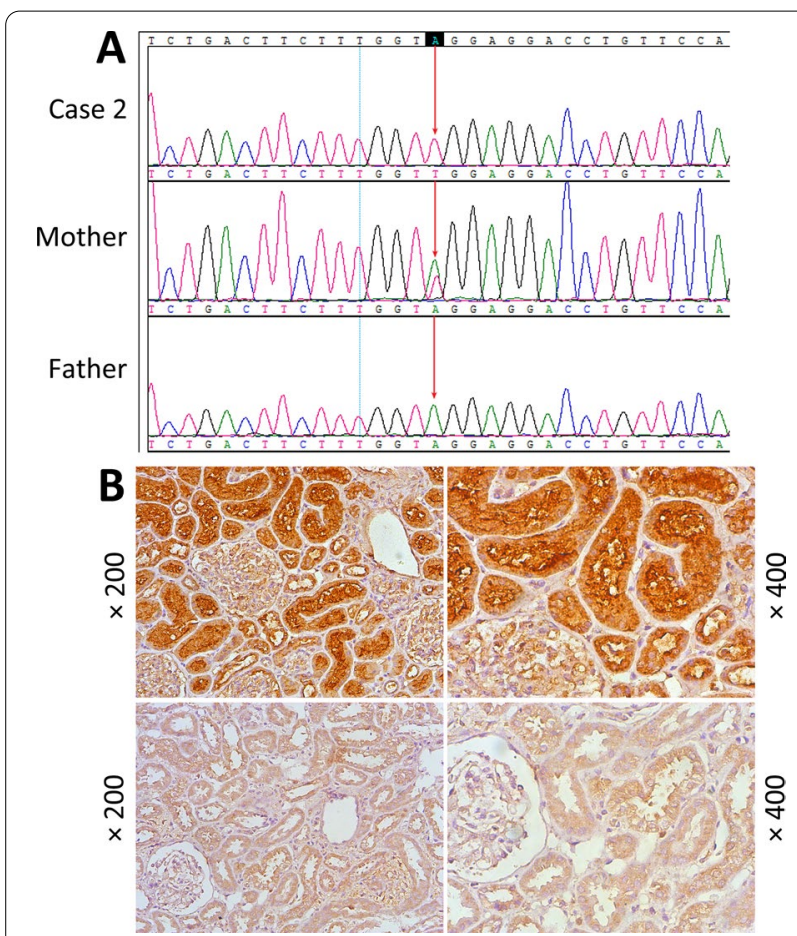

Fig. 4 OCRL mutation in patient 2. a DNA sequencing of the OCRL gene indicated a novel splicing mutation (hemizygous c.2257-2A>T), which was inherited from his mother. The IVS20 sequencing result for the mother indicated a heterozygous mutation. $\mathbf{b}$ Renal immunohistochemical staining of OCRL-1 in patient 2 and a control patient with thin basement membrane disease. Patient 2 had a significantly reduced OCRL-1 protein level compared to the control

\section{Discussion and conclusion}

The clinical diagnosis of Lowe syndrome is based on specific ophthalmologic, renal and neurologic abnormalities [8]. Bilateral cataract is a hallmark finding among all clinical features. A history of cataract surgery during the early infantile period provided an important clue for the diagnosis of Lowe syndrome in the patients. The neurological features of Lowe syndrome are muscle hypotonia, areflexia, intellectual disability, seizures, behavioral abnormalities (e.g., stubbornness, aggression, irritability, temper tantrums, complex repetitive purposeless movements, etc.), and abnormal findings in neuroimaging, such as brain atrophy, delayed myelination, mild ventriculomegaly, and hyperintense cerebral white matter on MRI T2-weighted images [9]. The two patients described here had the common clinical manifestations of muscle hypotonia, areflexia, and intellectual disability.

An MRI scan showed mild ventriculomegaly, bilateral periventricular leukomalacia, and multiple gliotic lesions in patient 1 , and brain atrophy, leukoaraiosis, and ventriculomegaly in patient 2 . Previous studies of affected patients reported the renal phenotype included LMW proteinuria, aminoaciduria, macroalbuminuria, hyperphosphaturia, hypercalciuria, glycosuria, metabolic acidosis, and progressive renal failure $[7,8]$. Renal tubular dysfunction is not always present at birth, but usually manifests within the first few weeks to months of life. The onset of renal tubulopathy can be asymptomatic, but can also manifest as Fanconi syndrome or even chronic renal failure, and disease severity tends to increase with age. LWM proteinuria is a prominent finding and occurs in all patients, although aminoaciduria, bicarbonaturia, and hypercalciuria are also frequently present [6]. Both of our patients had incomplete Fanconi syndrome. Thus, tubular proteinuria, hypercalciuria, phosphaturia, metabolic acidosis, and focal interstitial fibrosis were present, but other renal tubular functions were normal. We therefore consider our patients to have a severe phenotype of Lowe syndrome.

Clinical findings are considered adequate for the diagnosis of Lowe syndrome, but gene analysis is necessary for genetic counseling and analysis of genotype-phenotype correlation. According to the Human Gene Mutation Database (http://www.hgmd.cf.ac.uk/ac/index.php), there are approximately 250 pathogenic DNA variations of OCRL, and more than $90 \%$ are in exon $10-18$ and exon 19-23. Our patient 1 had a c.659_662delAGGG mutation in exon 8 , which caused a frameshift and a premature termination codon at aa 248. Patient 2 had one splice-site mutation in intron 20 , which presumably led to abnormal splicing of the RNA precursor and degradation of the mutated transcript by NMD. The two mutations identified here were not previously reported in any other 
patients with Lowe syndrome, and these two mutations seem related to a severe phenotype of Lowe syndrome.

To date, 30 OCRL mutations have been identified in Chinese patients with Lowe syndrome, including the two patients identified here (Table 2) [10-26]. Frameshift, splicing, or nonsense mutations leading to truncated proteins account for $67 \%$ of these mutations, missense mutations account for $27 \%$, and genomic deletions account for $6 \%$. Forty percent of these mutations occur in exons 9-16 (which encode the 5-phosphatase domain) and most of these mutations are single base pair missense changes. Another $40 \%$ of these mutations occur in exons 18-23 (which encode the RhoGAP-like domain in $\mathrm{C}$ terminus) and most of them cause truncated proteins. There was only one reported deletion mutation in exon 5 (which partially encodes the $\mathrm{PH}$ domain) and two reported mutations in exon
8 (which encodes the linkage domain between the $\mathrm{PH}$ and 5-phosphatase domains) that led to truncated proteins. Thus, based on literature review and our data, the genotype-phenotype correlation of OCRL mutations with the severe phenotype indicated clustering of frame-shifts and splicing mutations in or near the 5-phosphatase domain and the RhoGAP-like domain.

In summary, we identified two patients with novel $O C R L$ variants who had severe ocular and neurologic deficiency, although only mild renal dysfunction. This study also emphasizes that a genotype-phenotype correlation of $O C R L$ mutations with a severe phenotype of Lowe syndrome due to clustering of missense, deletion, and nonsense mutations in the 5-phosphatase domain and the Rho-GAP domain in the Chinese population.

Table 2 OCRL mutations in Chinese patients with Lowe syndrome

\begin{tabular}{|c|c|c|c|c|c|}
\hline $\begin{array}{l}\text { Patient } \\
\text { number }\end{array}$ & Mutation type & Exon/intron & Nucleotide change & Protein change & Literature \\
\hline 1 & Missense & Exon 21 & c.2290_2291delinsCT & p.Glu764Leu & Dai et al. [10] \\
\hline 2 & Missense & Exon 23 & c. $2581 G>A$ & p.Ala861Thr & Dai et al. [10] \\
\hline 3 & Missense & Exon 14 & c. $1423 C>T$ & p.Pro475Ser & Zheng et al. [11] \\
\hline 4 & Missense & Exon 15 & c. $1502 \mathrm{~T}>\mathrm{G}$ & p.lle501Ser & Zheng et al. [11] \\
\hline 5 & Nonsense & Exon 22 & c. $2464 C>T$ & p.Arg822Ter & Zheng et al. [11] \\
\hline 6 & Complex & Exon 22 & c.2368_2368delG, c.2370A >C & p.Ala790Profs*34 & Zhou et al. [12] \\
\hline 7 & Microdeletion (249 kb) & - & [hg19]arrXq25q26.1 $(128,652,372-128,901,629) \times 0$ & - & Zhang et al. [13] \\
\hline 8 & Deletion & Exon 14 & c.1389delT & p.Phe463Leufs*57 & Song et al. [14] \\
\hline 9 & Nonsense & Exon 11 & c. $1000 C>T$ & p.Arg334Ter & Gao et al. [15] \\
\hline 10 & Nonsense & Exon 18 & c. $2083 C>T$ & p.Arg695Ter & Gao et al. [15] \\
\hline 11 & Nonsense & Exon 8 & $\mathrm{c} .577 \mathrm{G}>\mathrm{T}$ & P.Glu193Ter & Zhang et al. [16] \\
\hline 12 & Microdeletion (633 kb) & - & [hg19] arrXq25q26.1 $(128,155,802-128,789,721) \times 0$ & - & Zhu et al. [17] \\
\hline 13 & Nonsense & Exon 15 & C. $1528 C>T$ & p.Gln510Ter & Gao et al. [18] \\
\hline 14 & Insertion & Exon 20 & c.2187dupG & p.Arg730Glufs*41 & Gao et al. [18] \\
\hline 15 & Nonsense & Exon 14 & c. $1366 C>T$ & p.Gln456Ter & Gao et al. [18] \\
\hline 16 & Missense & Exon 15 & C. $1499 G>A$ & p.Arg500Gln & Gao et al. [18] \\
\hline 17 & Deletion & Exon 13 & c.1281_1282delTT & p.Cys428Hisfs*2 & Li et al. [19] \\
\hline 18 & Insertion & Exon 22 & c.2367insA & p.Ala813Ter & Liu et al. [20] \\
\hline 19 & Splicing & Intron 20 & c. $2437+2 \_2437+4$ delTAAinsC & - & Ji et al. [21] \\
\hline 20 & Deletion & Exon 5 & c.321delC & p.Leu108Serfs*29 & Ji et al. [21] \\
\hline 21 & Complex & $\begin{array}{l}\text { Exon } 8 \\
\text { Exon } 22\end{array}$ & $\begin{array}{l}\text { c. } 562 C>T \\
\text { c. } 2464 C>T\end{array}$ & $\begin{array}{l}\text { p.Leu188Phe } \\
\text { p.Arg822Ter }\end{array}$ & Chen et al. [22] \\
\hline 22 & Missense & Exon 15 & c. $1571 A>G$ & p.His524Arg & Shi et al. [23] \\
\hline 23 & Deletion & Exon 18 & c.1897delT & p.Ser633Leufs*11 & Zhang et al. [24] \\
\hline 24 & Deletion & Exon 15 & c.1470delG & p.Lys491Asnfs*29 & Zhang et al. [24] \\
\hline 25 & Missense & Exon 15 & c. $1538 A>G$ & p.Tyr513Cys & Zhang et al. [24] \\
\hline 26 & Nonsense & Exon 10 & c.880G $>\mathrm{T}$ & p.Glu294Ter & Ke et al. [25] \\
\hline 27 & Insertion & Exon 24 & c.2626dupA & p.Met876Asnfs*8 & Ke et al. [25] \\
\hline 28 & Insertion & Exon 20 & c.2146_2147insTT & p.Ser716Phefs*27 & Chen et al. [26] \\
\hline 29 & Deletion & Exon 8 & c.659_662delAGGG & p.E220Vfs*29 & This report \\
\hline 30 & Splicing & Intron 20 & c. $2257-2 A>T$ & - & This report \\
\hline
\end{tabular}




\section{Abbreviations}

OCRL: Oculocerebrorenal syndrome of Lowe; PI(4,5)P2: Phosphatidylinositol 4,5-bisphosphate; HGMD: Human gene mutation database.

\section{Acknowledgements}

We thank the patients and relatives who participated in this study, and Dr. Yang $\mathrm{He}$ for assistance in developing the figure of the mutated OCRL-1 protein structure. This abstract was previously published in 2019 (https://www.kirep orts.org/article/S2468-0249(19)31345-2/fulltext).

\section{Authors' contributions}

$Z Y$ contributed to the conception of the study and wrote the manuscript. $\mathrm{HY}$ and DL collected data and performed the data analyses. ZY, CX, CY, and CK contributed to the management of the patients. ZJ helped perform the analysis with constructive discussions. All authors read and approved the final manuscript.

\section{Funding}

The authors received no financial support for the research, authorship, and/or publication of this article.

\section{Availability of data and materials}

The raw sequence data reported in this paper have been deposited in the Genome Sequence Archive (Genomics, Proteomics \& Bioinformatics 2017) in National Genomics Data Center (Nucleic Acids Res 2021), China National Center for Bioinformation/ Beijing Institute of Genomics, Chinese Academy of Sciences, under accession number HRA001169 that are publicly accessible at https://ngdc.cncb.ac.cn/gsa-human/browse/HRA001169.

\section{Declarations}

\section{Ethics approval and consent to participate}

This study was approved by the Ethical Committee of Tongji Hospital. Written informed consents were obtained from the parents of each pediatric patient (younger than 16 years old) for genetic and pathological investigation. Written informed consents were also obtained from each adult participant for genetic investigation.

\section{Consent for publication}

The parents of each patient provided written consents for the publication of this study. Written consent documents regarding photographs, clinical and genetic details have been obtained from the parents of each patient.

\section{Competing interests}

The authors declare no competing interests.

\section{Author details}

1'Department of Pediatrics, Tongji Hospital, Tongji Medical College, Huazhong University of Science and Technology, No.1095, Jiefang Ave., Wuhan 430030, People's Republic of China. ${ }^{2}$ Department of Endocrinology and Metabolism, Wuhan Children's Hospital, Tongji Medical College, Huazhong University of Science and Technology, Wuhan, China.

Received: 28 December 2020 Accepted: 27 August 2021

Published online: 06 September 2021

\section{References}

1. Lowe CU, Terrey M, MacLachlan EA. Organic-aciduria, decreased renal ammonia production, hydrophthalmos, and mental retardation; a clinical entity. AMA Am J Dis Child. 1952;83(2):164-84.

2. Loi M. Lowe syndrome. Orphanet J Rare Dis. 2006;1 (1):16.

3. Zaniew M, Bokenkamp A, Kolbuc M, La SC, Baronio F, Niemirska A, Szczepanska M, Bürger J, La MA, Miklaszewska M, et al. Long-term renal outcome in children with OCRL mutations: retrospective analysis of a large international cohort. Nephrol Dial Transpl. 2018;33(1):85-94.
4. Zhang X, Jefferson AB, Auethavekiat V, Majerus PW. The protein deficient in Lowe syndrome is a phosphatidylinositol-4,5-bisphosphate 5-phosphatase. Proc Natl Acad Sci USA. 1995;92(11):4853-6.

5. Suchy SF, Olivos-Glander IM, Nussbaum RL. Lowe syndrome, a deficiency of a phosphatidylinositol 4,5-bisphosphate 5-phosphatase in the Golgi apparatus. Hum Mol Genet. 1995;4(12):2245-50.

6. Mehta ZB, Pietka G, Lowe M. The cellular and physiological functions of the lowe syndrome protein OCRL1. Traffic. 2014;15(5):471-87.

7. Hichri H, Rendu J, Monnier N, Coutton C, Dorseuil O, Poussou R, Baujat G, Blanchard A, Nobili F, Ranchin B, et al. From Lowe syndrome to dent disease: correlations between mutations of the OCRL1 gene and clinical and biochemical phenotypes. Hum Mutat. 2011;32(4):379-88.

8. Bokenkamp A, Ludwig M. The oculocerebrorenal syndrome of Lowe: an update. Pediatr Nephrol. 2016:31(12):2201-12.

9. Allmendinger AM, Desai NS, Burke AT, Viswanadhan N, Prabhu S. Neuroimaging and renal ultrasound manifestations of Oculocerebrorenal syndrome of Lowe. J Radiol Case Rep. 2014;8(10):1-7.

10. Dai C, Wang L, Li Y, Zheng Z, Qian J, Wang C, Liu Z, Shan X. Lowe syndrome with extremely short stature: growth hormone deficiency may be the pathogeny. Growth Factors. 2019:37(3-4):170-7.

11. Zheng B, Chen Q, Wang C, Zhou W, Chen Y, Ding G, Jia Z, Zhang A, Huang S. Whole-genome sequencing revealed an interstitial deletion encompassing OCRL and SMARCA1 gene in a patient with Lowe syndrome. Mol Genet Genomic Med. 2019;7(9):e876.

12. Zhou FQ, Wang QW, Liu ZZ, Zhang XL, Wang DN, Dongye MM, Lin HT, Chen WR. Novel mutation in OCRL leading to a severe form of Lowe syndrome. Int J Ophthalmol-Chi. 2019;12(7):1057-60.

13. Zhang $Y$, Li R, Jing X, Tang X, Li F, Liao C. Clinical and molecular genetic analysis of a pediatric patient with Lowe syndrome. Zhonghua Yi Xue Yi Chuan Xue Za Zhi. 2019;36(6):613-5.

14. Song Y, Li G. A case of infant lowe syndrome. Shangdong Da Xue Xue Bao (Yi Xue Ban). 2019;57(4):119-21.

15. Gao S, Li J, Lin J. Two cases of children lowe syndrome. Sichuang Da Xue Xue Bao (Yi Xue Ban). 2019;50(1):144.

16. Zhang C, Wang S, Chen R, Zheng L, Liao Z. A case of oculo-cerebro-renal syndrome in adult. Zhongguo Shen Jing Mian YI Xue He Shen Jing Bing Xue Za Zhi. 2017;24(3):225-6.

17. Zhu X, Li J, Ru T, Zhu RF, Dai CY, Wang WJ, Hu YL. Prenatal diagnosis and follow-up of a case with Lowe syndrome caused by interstitial deletion of Xq25-26. Zhonghua Yi Xue Yi Chuan Xue Za Zhi. 2017;34(2):236-9.

18. Gao Y, Jiang F, Ou ZY. Novel OCRL1 gene mutations in six Chinese families with Lowe syndrome. World J Pediatr. 2016:12(4):484-8.

19. Li B, Zhang Z, Zhou Q, Yang J, Wu X, Liu G. Clinical features and OCRL mutation analysis in a case of infant Lowe syndrome. Chin J Pathophysiol. 2016;32(7):1302-6.

20. Liu T, Yue Z, Wang H, Tong H, Sun L. Novel mutation of OCRL1 in Lowe syndrome. Indian J Pediatr. 2015;82(1):89-92

21. Ji L, Chen C, Li H, Du P. Lowe syndrome with novel OCRL mutations in Chinese children: report of two cases. J Clin Pediatrics. 2015;33(6):531-4.

22. Chen $\mathrm{Q}$, Chen Y, Zhang W. A case of Lowe syndrome. Zhonghua Er Ke Za Zhi. 2015;53(11):862-3.

23. Shi RM, Bian XH, Li LM, Liu XH. Investigation and OCRL mutation analysis of a family with oculocerebrorenal syndrome of Lowe. Zhongguo Dang Dai Er Ke Za Zhi. 2014;16(4):366-9.

24. Zhang YQ, Wang F, Ding J, Yan H, Yang YL. Novel OCRL mutations in Chinese children with Lowe syndrome. World J Pediatr. 2013;9(1):53-7.

25. Ke YH, He JW, Fu WZ, Zhang ZL. Identification of two novel mutations in the OCRL1 gene in two Chinese families with Lowe syndrome. Nephrology (Carlton). 2012;17(1):20-5.

26. Chen S, Zhang X, Chen L, Tian Q, Jiang W. Analysis of OCRL gene mutation in a male infant with Lowe syndrome. Zhonghua Yi Xue Yi Chuan Xue Za Zhi. 2014:31(2):223-7.

\section{Publisher's Note}

Springer Nature remains neutral with regard to jurisdictional claims in published maps and institutional affiliations. 